\title{
Minimal complex surfaces with Levi-Civita Ricci-flat metrics
}

\author{
Kefeng Liu and Xiaokui Yang \\ In memory of Professor Lu Qi-Keng
}

\begin{abstract}
This is a continuation of our previous paper [14]. In [14], we introduced the first Aeppli-Chern class on compact complex manifolds, and proved that the $(1,1)$ curvature form of the Levi-Civita connection represents the first Aeppli-Chern class which is a natural link between Riemannian geometry and complex geometry. In this paper, we study the geometry of compact complex manifolds with Levi-Civita Ricci-flat metrics and classify minimal complex surfaces with LeviCivita Ricci-flat metrics. More precisely, we show that minimal complex surfaces admitting Levi-Civita Ricci-flat metrics are Kähler Calabi-Yau surfaces and Hopf surfaces.
\end{abstract}

\section{Contents}

1. Introduction 1

2. Preliminaries 3

3. The Levi-Civita connection on the holomorphic tangent bundle 4

4. Geometry of the first Aeppli-Chern class 6

5. Compact complex manifolds with Levi-Civita Ricci-flat metrics 7

6. Classification of minimal complex surfaces with Levi-Civita Ricci-flat metrics 9

7. The Levi-Civita Ricci-flat metrics on minimal surfaces of class VII 11

$\begin{array}{ll}\text { References } & 13\end{array}$

\section{Introduction}

In this paper, we study the relationship between Riemannian manifolds and complex manifolds by using various metric connections and their curvature tensors.

Let $(X, h)$ be a Hermitian manifold and $g$ be the background Riemannian metric. It is well-known that, when $(X, h)$ is not Kähler, the relation between the Riemannian geometry $(X, g)$ and the complex geometry $(X, h)$ is extremely complicated. Indeed, on the Hermitian holomorphic tangent bundle $\left(T^{1,0} X, h\right)$, there are two typical metric compatible connections:

(1) the Chern connection $\nabla$, i.e. the unique connection $\nabla$ compatible with the Hermitian metric and also the complex structure $\bar{\partial}$;

(2) the Levi-Civita connection $\nabla^{\mathrm{LC}}$, i.e. the restriction of the complexified Levi-Civita connection on $T_{\mathbb{C}} X$ to the holomorphic tangent bundle $T^{1,0} X$.

From the definition, it is quite obvious that the Levi-Civita connection $\nabla^{\mathrm{LC}}$ is a representative of the Riemannian geometry of $(X, g)$. It is also well-known that when $(X, h)$ is not Kähler, 
$\nabla$ and $\nabla^{\mathrm{LC}}$ are not the same. The complex geometry of the Chern connection is extensively investigated in the literatures by using various methods (e.g. [3, 7, 8, 9, 12, 13, 14, 15, 16, 17, $19,20,21,22,23,24,25])$. However, the complex geometry of the Levi-Civita connection is not well understood although it has rich Riemannian geometry structures..

In [14], we introduced the first Aeppli-Chern classes for holomorphic line bundles. Let $L \rightarrow X$ be a holomorphic line bundle over $X$. The first Aeppli-Chern class is defined as

$$
c_{1}^{\mathrm{AC}}(L)=[-\sqrt{-1} \partial \bar{\partial} \log h]_{\mathrm{A}} \in H_{\mathrm{A}}^{1,1}(X)
$$

where $h$ is an arbitrary smooth Hermitian metric on $L$ and the Aeppli cohomology is

$$
H_{\mathrm{A}}^{p, q}(X):=\frac{\operatorname{Ker} \partial \bar{\partial} \cap \Omega^{p, q}(X)}{\operatorname{Im} \partial \cap \Omega^{p, q}(X)+\operatorname{Im} \bar{\partial} \cap \Omega^{p, q}(X)} .
$$

For a complex manifold $X, c_{1}^{\mathrm{AC}}(X)$ is defined to be $c_{1}^{\mathrm{AC}}\left(K_{X}^{-1}\right)$ where $K_{X}^{-1}$ is the anti-canonical line bundle of $X$. Note that, for a Hermitian line bundle $(L, h)$, the classes $c_{1}(L)$ and $c_{1}^{\mathrm{AC}}(L)$ have the same $(1,1)$-form representative $\Theta^{h}=-\sqrt{-1} \partial \bar{\partial} \log h$ (in different classes). It is well-known that on a Hermitian manifold $(X, \omega)$, the first Chern-Ricci curvature $\operatorname{Ric}(\omega)=$ $-\sqrt{-1} \partial \bar{\partial} \log \operatorname{det}(\omega)$ represents the first Chern class $c_{1}(X)$. As an analog, we proved in [14, Theorem1.1] that the first Levi-Civita Ricci curvature $\mathfrak{R i c}(\omega)$ represents the first Aeppli-Chern class $c_{1}^{\mathrm{AC}}(X)$. It is obvious that $c_{1}(X)=0$ implies $c_{1}^{\mathrm{AC}}(X)=0$. Hence, it is very natural to study non-Kähler Calabi-Yau manifolds by using the first Aeppli-Chern class $c_{1}^{\mathrm{AC}}(X)$ and the first Levi-Civita Ricci curvature $\mathfrak{R i c}(\omega)$. By the celebrated Calabi-Yau theorem ([26]), a compact Kähler manifold has $c_{1}(X)=0$ if and only if it has a Kähler metric with Ricci-flat metric, i.e. $\operatorname{Ric}(\omega)=0$. It is easy to see that if $\mathfrak{R i c}(\omega)=0$, then $c_{1}^{\mathrm{AC}}(X)=0$. There is a natural question analogous to the Calabi conjecture:

Question 1.1. On a compact complex manifold $X$, if $c_{1}^{\mathrm{AC}}(X)=0$, does there exist a smooth Levi-Civita Ricci-flat Hermitian metric $\omega$, i.e. $\mathfrak{R i c}(\omega)=0$ ?

As we have shown in [14, Theorem 1.2], $\mathfrak{R i c}(\omega)=0$ is equivalent to

$$
\operatorname{Ric}(\omega)=\frac{1}{2}\left(\partial \partial^{*} \omega+\overline{\partial \partial}^{*} \omega\right) .
$$

The equation (1.2) is not the Monge-Ampère type equation since there are also non-elliptic second order derivatives on the right hand side. As it is well-known, it is particularly challenging to solve such equations. Instead of solving the equation (1.2) directly, we use several observations in [24] to study the geometry of the equation (1.2) and obtain necessary conditions to solve (1.2). See Corollary 5.1, Theorem 5.2 and Theorem 5.4 in Section 5 for more details. By using these necessary conditions and Kodaira-Enriques' classification (e.g. [2, p. 244]) of minimal complex surfaces, we obtain:

Theorem 1.2. Let $X$ be a minimal complex surface. Suppose $X$ admits a Levi-Civita Ricciflat Hermitian metric $\omega$. Then $X$ lies in one of the following

(1) Enriques surfaces;

(2) bi-elliptic surfaces;

(3) K3 surfaces;

(4) 2-tori;

(5) Hopf surfaces. 
It worths to point out that we do not show every Hopf surface admitting a Levi-Civita Ricciflat metric. We only construct such metrics on diagonal Hopf surfaces (see Theorem 7.3). We conjecture that all Hopf surfaces can support Levi-Civita Ricci-flat metrics. On the other hand, every Hopf surface $X$ has $c_{1}(X)=0 \in H^{2}(X, \mathbb{R})$. However, it is easy to show that $X$ can not support Chern-Ricci flat Hermitian metrics, i.e. Hermitian metrics $\omega$ with $\operatorname{Ric}(\omega)=0$.

As an application of Theorem 1.2, we obtain the following example which indicates that we need extra constraints to solve Question 1.1 in general:

Corollary 1.3. Let $X$ be a Kodaira surface or an Inoue surface. Then

$$
c_{1}(X)=c_{1}^{\mathrm{BC}}(X)=c_{1}^{\mathrm{AC}}(X)=0 .
$$

However, $X$ does not admit a Levi-Civita Ricci-flat Hermitian metric.

Acknowledgement. The second author would like to thank Valentino Tosatti for many useful comments and suggestions. This work was partially supported by China's Recruitment Program of Global Experts, National Center for Mathematics and Interdisciplinary Sciences, Chinese Academy of Sciences.

\section{Preliminaries}

2.1. Chern connection on complex manifolds. Let $\left(X, \omega_{g}\right)$ be a compact Hermitian manifold. There exists a unique connection $\nabla$ on the holomorphic tangent bundle $T^{1,0} X$ which is compatible with the Hermitian metric and also the complex structure. This connection $\nabla$ is called the Chern connection. The Chern connection $\nabla$ on $\left(T^{1,0} X, \omega_{g}\right)$ has curvature components

$$
R_{i \bar{j} k \bar{\ell}}=-\frac{\partial^{2} g_{k \bar{\ell}}}{\partial z^{i} \partial \bar{z}^{j}}+g^{p \bar{q}} \frac{\partial g_{k \bar{q}}}{\partial z^{i}} \frac{\partial g_{p \bar{\ell}}}{\partial \bar{z}^{j}} .
$$

The (first) Chern-Ricci form $\operatorname{Ric}\left(\omega_{g}\right)$ of $\left(X, \omega_{g}\right)$ has components

$$
R_{i \bar{j}}=g^{k \bar{\ell}} R_{i \bar{j} k \bar{\ell}}=-\frac{\partial^{2} \log \operatorname{det}(g)}{\partial z^{i} \partial \bar{z}^{j}}
$$

which also represents the first Chern class $c_{1}(X)$ of the complex manifold $X$. The Chern scalar curvature $s_{g}$ of $\left(X, \omega_{g}\right)$ is given by

$$
s_{g}=\operatorname{tr}_{\omega_{g}} \operatorname{Ric}\left(\omega_{g}\right)=g^{i \bar{j}} R_{i \bar{j}} .
$$

The total scalar curvature of $\omega_{g}$ is

$$
\int_{X} s_{g} \omega_{g}^{n}=n \int \operatorname{Ric}\left(\omega_{g}\right) \wedge \omega_{g}^{n-1},
$$

where $n$ is the complex dimension of $X$. 
2.2. Bott-Chern classes and Aeppli classes. The Bott-Chern cohomology and the Aeppli cohomology on a compact complex manifold $X$ are given by

$$
H_{\mathrm{BC}}^{p, q}(X):=\frac{\operatorname{Ker} d \cap \Omega^{p, q}(X)}{\operatorname{Im} \partial \bar{\partial} \cap \Omega^{p, q}(X)} \quad \text { and } \quad H_{\mathrm{A}}^{p, q}(X):=\frac{\operatorname{Ker} \partial \bar{\partial} \cap \Omega^{p, q}(X)}{\operatorname{Im} \partial \cap \Omega^{p, q}(X)+\operatorname{Im} \bar{\partial} \cap \Omega^{p, q}(X)} .
$$

Let $\operatorname{Pic}(X)$ be the set of holomorphic line bundles over $X$. As similar as the first Chern class map $c_{1}: \operatorname{Pic}(X) \rightarrow H_{\bar{\partial}}^{1,1}(X)$, there is a first Aeppli-Chern class map

$$
c_{1}^{\mathrm{AC}}: \operatorname{Pic}(X) \rightarrow H_{\mathrm{A}}^{1,1}(X) .
$$

Given any holomorphic line bundle $L \rightarrow X$ and any Hermitian metric $h$ on $L$, its curvature form $\Theta_{h}$ is locally given by $-\sqrt{-1} \partial \bar{\partial} \log h$. We define $c_{1}^{\mathrm{AC}}(L)$ to be the class of $\Theta_{h}$ in $H_{\mathrm{A}}^{1,1}(X)$. For a complex manifold $X, c_{1}^{\mathrm{AC}}(X)$ is defined to be $c_{1}^{\mathrm{AC}}\left(K_{X}^{-1}\right)$ where $K_{X}^{-1}$ is the anti-canonical line bundle. The first Bott-Chern class $c_{1}^{\mathrm{BC}}(X)$ can be defined similary.

2.3. Special manifolds. Let $X$ be a compact complex manifold.

(1) A Hermitian metric $\omega_{g}$ is called a Gauduchon metric if $\partial \bar{\partial} \omega_{g}^{n-1}=0$. It is proved by Gauduchon ([10]) that, in the conformal class of each Hermitian metric, there exists a unique Gauduchon metric (up to scaling).

(2) A Hermitian metric $\omega_{g}$ is called a balanced metric if $d \omega_{g}^{n-1}=0$ or equivalently $d^{*} \omega_{g}=0$. On a compact complex surface, a balanced metric is also Kähler, i.e. $d \omega_{g}=0$. It is well-known many Hermitian manifolds can not support balanced metrics, e.g. Hopf surface $\mathbb{S}^{3} \times \mathbb{S}^{1}$. It is also obvious that balanced metrics are Gauduchon.

(3) $X$ is called a Calabi-Yau manifold if $c_{1}(X)=0 \in H^{2}(X, \mathbb{R})$.

It is obvious that,

$$
c_{1}^{\mathrm{BC}}(X)=0 \Longrightarrow c_{1}(X)=0 \Longrightarrow c_{1}^{\mathrm{AC}}(X)=0,
$$

and on compact Kähler manifolds or manifolds supporting the $\partial \bar{\partial}$-lemma ([14, Corollary 1.4]), they are equivalent.

\section{The Levi-Civita connection on the holomorphic tangent bundle}

Let's recall some elementary settings (e.g. [14, Section 2]). Let $(M, g, \nabla)$ be a $2 n$ dimensional Riemannian manifold with the Levi-Civita connection $\nabla$. The tangent bundle of $M$ is also denoted by $T_{\mathbb{R}} M$. The Riemannian curvature tensor of $(M, g, \nabla)$ is

$$
R(X, Y, Z, W)=g\left(\nabla_{X} \nabla_{Y} Z-\nabla_{Y} \nabla_{X} Z-\nabla_{[X, Y]} Z, W\right)
$$

for tangent vectors $X, Y, Z, W \in T_{\mathbb{R}} M$. Let $T_{\mathbb{C}} M=T_{\mathbb{R}} M \otimes \mathbb{C}$ be the complexification. We can extend the metric $g$ and the Levi-Civita connection $\nabla$ to $T_{\mathbb{C}} M$ in the $\mathbb{C}$-linear way. Hence for any $a, b, c, d \in \mathbb{C}$ and $X, Y, Z, W \in T_{\mathbb{C}} M$, we have

$$
R(a X, b Y, c Z, d W)=a b c d \cdot R(X, Y, Z, W) .
$$

Let $(M, g, J)$ be an almost Hermitian manifold, i.e., $J: T_{\mathbb{R}} M \rightarrow T_{\mathbb{R}} M$ with $J^{2}=-1$, and for any $X, Y \in T_{\mathbb{R}} M, g(J X, J Y)=g(X, Y)$. The Nijenhuis tensor $N_{J}: \Gamma\left(M, T_{\mathbb{R}} M\right) \times$ 
$\Gamma\left(M, T_{\mathbb{R}} M\right) \rightarrow \Gamma\left(M, T_{\mathbb{R}} M\right)$ is defined as

$$
N_{J}(X, Y)=[X, Y]+J[J X, Y]+J[X, J Y]-[J X, J Y] .
$$

The almost complex structure $J$ is called integrable if $N_{J} \equiv 0$ and then we call $(M, g, J)$ a Hermitian manifold. We can also extend $J$ to $T_{\mathbb{C}} M$ in the $\mathbb{C}$-linear way. Hence for any $X, Y \in T_{\mathbb{C}} M$, we still have $g(J X, J Y)=g(X, Y)$. By Newlander-Nirenberg's theorem, there exists a real coordinate system $\left\{x^{i}, x^{I}\right\}$ such that $z^{i}=x^{i}+\sqrt{-1} x^{I}$ are local holomorphic coordinates on $M$. Moreover, we have $T_{\mathbb{C}} M=T^{1,0} M \oplus T^{0,1} M$ where

$$
T^{1,0} M=\operatorname{span}_{\mathbb{C}}\left\{\frac{\partial}{\partial z^{1}}, \cdots, \frac{\partial}{\partial z^{n}}\right\} \quad \text { and } \quad T^{0,1} M=\operatorname{span}_{\mathbb{C}}\left\{\frac{\partial}{\partial \bar{z}^{1}}, \cdots, \frac{\partial}{\partial \bar{z}^{n}}\right\} .
$$

Since $T^{1,0} M$ is a subbundle of $T_{\mathbb{C}} M$, there is an induced connection $\nabla^{\mathrm{LC}}$ on the holomorphic tangent bundle $T^{1,0} M$ given by

$$
\nabla^{\mathrm{LC}}=\pi \circ \nabla: \Gamma\left(M, T^{1,0} M\right) \stackrel{\nabla}{\rightarrow} \Gamma\left(M, T_{\mathbb{C}} M \otimes T_{\mathbb{C}} M\right) \stackrel{\pi}{\rightarrow} \Gamma\left(M, T_{\mathbb{C}} M \otimes T^{1,0} M\right) .
$$

Let $h=\left(h_{i \bar{j}}\right)$ be the corresponding Hermitian metric on $T^{1,0} M$ induced by $(M, g, J)$. It is obvious that $\nabla^{\mathrm{LC}}$ is a metric compatible connection on the Hermitian holomorphic vector bundle $\left(T^{1,0} M, h\right)$, and we call $\nabla^{\mathrm{LC}}$ the Levi-Civita connection on the complex manifold $M$. It is obvious that, $\nabla^{\mathrm{LC}}$ is determined by the following relations

$$
\nabla_{\frac{\partial}{\partial z^{2}}}^{\mathrm{LC}} \frac{\partial}{\partial z^{k}}:=\Gamma_{i k}^{p} \frac{\partial}{\partial z^{p}} \quad \text { and } \quad \nabla_{\frac{\partial}{\partial \bar{z}^{j}}}^{\mathrm{LC}} \frac{\partial}{\partial z^{k}}:=\Gamma_{\bar{j} k}^{p} \frac{\partial}{\partial z^{p}}
$$

where

$$
\Gamma_{i j}^{k}=\frac{1}{2} h^{k \bar{\ell}}\left(\frac{\partial h_{j \bar{\ell}}}{\partial z^{i}}+\frac{\partial h_{i \bar{\ell}}}{\partial z^{j}}\right), \quad \text { and } \quad \Gamma_{\bar{i} j}^{k}=\frac{1}{2} h^{k \bar{\ell}}\left(\frac{\partial h_{j \bar{\ell}}}{\partial \bar{z}^{i}}-\frac{\partial h_{j \bar{i}}}{\partial \bar{z}^{\ell}}\right) .
$$

The curvature tensor $\mathfrak{R} \in \Gamma\left(M, \Lambda^{2} T_{\mathbb{C}} M \otimes T^{* 1,0} M \otimes T^{1,0} M\right)$ of $\nabla^{\mathrm{LC}}$ is given by

$$
\mathfrak{R}(X, Y) s=\nabla_{X}^{\mathrm{LC}} \nabla_{Y}^{\mathrm{LC}} s-\nabla_{Y}^{\mathrm{LC}} \nabla_{X}^{\mathrm{LC}} s-\nabla_{[X, Y]}^{\mathrm{LC}} s
$$

for any $X, Y \in T_{\mathbb{C}} M$ and $s \in T^{1,0} M$. A straightforward computation shows that the curvature tensor $\mathfrak{R}$ has $(1,1)$ components

$$
\mathfrak{R}_{i \bar{j} k}^{\ell}=-\left(\frac{\partial \Gamma_{i k}^{\ell}}{\partial \bar{z}^{j}}-\frac{\partial \Gamma_{\bar{j} k}^{\ell}}{\partial z^{i}}+\Gamma_{i k}^{s} \Gamma_{\bar{j} s}^{\ell}-\Gamma_{\bar{j} k}^{s} \Gamma_{s i}^{\ell}\right) .
$$

Definition 3.1. The (first) Levi-Civita Ricci curvature $\mathfrak{R i c}\left(\omega_{h}\right)$ of the Hermitian vector bundle $\left(T^{1,0} M, \omega_{h}, \nabla^{\mathrm{LC}}\right)$ is

$$
\mathfrak{R i c}\left(\omega_{h}\right)=\sqrt{-1} \mathfrak{R}_{i \bar{j}}^{(1)} d z^{i} \wedge d \bar{z}^{j} \quad \text { with } \quad \mathfrak{R}_{i \bar{j}}^{(1)}=\mathfrak{R}_{i \bar{j} k}^{k} .
$$

The Levi-Civita scalar curvature $s_{\mathrm{LC}}$ of $\nabla^{\mathrm{LC}}$ on $T^{1,0} M$ is

$$
s_{\mathrm{LC}}=h^{i \bar{j}} h^{k \bar{\ell}} \mathfrak{R}_{i \bar{j} k \bar{\ell}} .
$$


4. Geometry of the first Aeppli-Chern class

Let's give a straightforward proof of [14, Theorem 1.2].

Theorem 4.1. Let $(X, \omega)$ be a compact Hermitian manifold. Then the first Levi-Civita Ricci form $\mathfrak{R i c}(\omega)$ represents the first Aeppli-Chern class $c_{1}^{\mathrm{AC}}(X)$ in $H_{\mathrm{A}}^{1,1}(X)$. Moreover, we have the Ricci curvature relation

$$
\mathfrak{R i c}(\omega)=\operatorname{Ric}(\omega)-\frac{1}{2}\left(\partial \partial^{*} \omega+\overline{\partial \partial}^{*} \omega\right),
$$

and the scalar curvature relation

$$
s_{\mathrm{LC}}=s_{\mathrm{C}}-\left\langle\partial \partial^{*} \omega, \omega\right\rangle .
$$

Proof. It is easy to show that

$$
\bar{\partial}^{*} \omega=2 \sqrt{-1} \overline{\Gamma_{\bar{i} k}^{k}} d z^{i}
$$

and so

$$
-\frac{\partial \partial^{*} \omega+\overline{\partial \partial}^{*} \omega}{2}=\sqrt{-1}\left(\frac{\partial \Gamma \frac{k}{j} k}{\partial z^{i}}+\frac{\partial \overline{\Gamma_{\bar{i} k}^{k}}}{\partial \bar{z}^{j}}\right) d z^{i} \wedge d \bar{z}^{j} .
$$

On the other hand, by formula (3.4), we have

$$
\Re_{i \bar{j}}=\Re_{i \bar{j} k}^{k}=-\frac{\partial \Gamma_{i k}^{k}}{\partial \bar{z}^{j}}+\frac{\partial \Gamma_{\bar{j} k}^{k}}{\partial z^{i}}
$$

Moreover, we have

$$
\left(-\frac{\partial \Gamma_{i k}^{k}}{\partial \bar{z}^{j}}+\frac{\partial \Gamma_{\bar{j} k}^{k}}{\partial z^{i}}\right)-\left(\frac{\partial \Gamma_{\bar{j} k}^{k}}{\partial z^{i}}+\frac{\partial \overline{\Gamma_{\bar{i} k}^{k}}}{\partial \bar{z}^{j}}\right)=-\frac{\partial \Gamma_{i k}^{k}}{\partial \bar{z}^{j}}-\frac{\partial \overline{\Gamma_{\bar{i} k}^{k}}}{\partial \bar{z}^{j}}=-\frac{\partial^{2} \log \operatorname{det}(g)}{\partial z^{i} \partial \bar{z}^{j}}
$$

which establishes formula (4.1). It is easy to show $\left\langle\partial \partial^{*} \omega, \omega\right\rangle=\left\langle\overline{\partial \partial}^{*} \omega, \omega\right\rangle$.

Lemma 4.2. Let $(X, \omega)$ be a compact Hermitian manifold with complex dimension $n$. Suppose $f \in C^{\infty}(X, \mathbb{R})$ and $\omega_{f}=e^{f} \omega$. Then we have

$$
\bar{\partial}_{f}^{*} \omega_{f}=\bar{\partial}^{*} \omega+\sqrt{-1}(n-1) \partial f \quad \text { and } \quad \overline{\partial \partial}_{f}^{*} \omega_{f}=\overline{\partial \partial}^{*} \omega-\sqrt{-1}(n-1) \partial \bar{\partial} f .
$$

where $\bar{\partial}^{*}, \bar{\partial}_{f}^{*}$ are the adjoint operators with respect to the metric $\omega$ and $\omega_{f}$ respectively.

Definition 4.3. The Kodaira dimension $\kappa(L)$ of a line bundle $L$ is defined to be

$$
\kappa(L):=\limsup _{m \rightarrow+\infty} \frac{\log \operatorname{dim}_{\mathbb{C}} H^{0}\left(X, L^{\otimes m}\right)}{\log m}
$$

and the Kodaira dimension $\kappa(X)$ of $X$ is defined as $\kappa(X):=\kappa\left(K_{X}\right)$ where the logarithm of zero is defined to be $-\infty$.

Theorem 4.4. Let $(X, \omega)$ be a compact Hermitian manifold. Suppose the Levi-Civita scalar curvature $s_{\mathrm{LC}}$ of $\omega$ is positive, then $K_{X}$ is not pseudo-effective and $\kappa(X)=-\infty$. 
Proof. Let $\omega_{f}=e^{f} \omega$ be the Gauduchon metric in the conformal class of $\omega$. Then by Lemma 4.2 and Theorem 4.1, we have

$$
\begin{aligned}
\operatorname{Ric}\left(\omega_{f}\right)-\frac{\partial \partial_{f}^{*} \omega_{f}+\overline{\partial \partial}_{f}^{*} \omega_{f}}{2} & =\operatorname{Ric}(\omega)-\frac{\partial \partial^{*} \omega+\overline{\partial \partial}^{*} \omega}{2}-\sqrt{-1} \partial \bar{\partial} f \\
& =\operatorname{Ric}(\omega)-\sqrt{-1} \partial \bar{\partial} f .
\end{aligned}
$$

Moreover, we have

$$
\begin{aligned}
\int_{X} \operatorname{Ric}\left(\omega_{f}\right) \wedge \omega_{f}^{n-1} & =\int_{X}\left(\mathfrak{R i c}(\omega)-\sqrt{-1} \partial \bar{\partial} f+\frac{\partial \partial_{f}^{*} \omega_{f}+\overline{\partial \partial}^{*} \omega_{f}}{2}\right) \wedge \omega_{f}^{n-1} \\
& =\int_{X} \Re \mathfrak{i c}(\omega) \wedge \omega_{f}^{n-1}+\frac{1}{2}\left(\left\|\bar{\partial}_{f}^{*} \omega_{f}\right\|_{\omega_{f}}^{2}+\left\|\partial_{f}^{*} \omega_{f}\right\|_{\omega_{f}}^{2}\right) \\
& =\frac{1}{n} \int_{X} e^{(n-1) f} \cdot s_{\mathrm{LC}} \cdot \omega^{n}+\frac{1}{2}\left(\left\|\bar{\partial}_{f}^{*} \omega_{f}\right\|_{\omega_{f}}^{2}+\left\|\partial_{f}^{*} \omega_{f}\right\|_{\omega_{f}}^{2}\right) .
\end{aligned}
$$

Suppose the Levi-Civita scalar curvature $s_{\mathrm{LC}}>0$, then the total Chern scalar curvature of the Gauduchon metric $\omega_{f}$ is strictly positive, i.e.

$$
\int_{X} s_{f} \cdot \omega_{f}^{n}=n \int_{X} \operatorname{Ric}\left(\omega_{f}\right) \wedge \omega_{f}^{n-1}>0 .
$$

By [24, Theorem 1.1] and [24, Corollary 3.3], we know $K_{X}$ is not pseudo-effective and $\kappa(X)=$ $-\infty$.

Corollary 4.5. Let $X$ be a compact complex manifold with $c_{1}^{\mathrm{BC}}(X)=0$, then there is no Hermitian metric with positive Levi-Civita scalar curvature.

Proof. If $c_{1}^{\mathrm{BC}}(X)=0$, then by [17, Theorem 1.3], there exists a smooth Gauduchon metric $\omega_{g}$ with $\operatorname{Ric}\left(\omega_{g}\right)=0$. Hence for any other Gauduchon metric $\omega_{G}$, we have

$$
\int_{X} \operatorname{Ric}\left(\omega_{G}\right) \wedge \omega_{G}^{n-1}=0
$$

Suppose $\omega$ is a Hermitian metric with positive Levi-Civita scalar curvature $s_{\mathrm{LC}}$ and $\omega_{f}$ is the Gauduchon metric in the conformal class of $\omega$, then by (4.8), we have

$$
\int_{X} \operatorname{Ric}\left(\omega_{f}\right) \wedge \omega_{f}^{n-1}=\frac{1}{n} \int_{X} e^{(n-1) f} \cdot s_{\mathrm{LC}} \cdot \omega^{n}+\frac{1}{2}\left(\left\|\bar{\partial}_{f}^{*} \omega_{f}\right\|_{\omega_{f}}^{2}+\left\|\partial_{f}^{*} \omega_{f}\right\|_{\omega_{f}}^{2}\right)>0,
$$

which is a contradiction.

\section{Compact complex manifolds with Levi-Civita Ricci-flat metrics}

Let's recall that, a Levi-Civita Ricci-flat metric is a Hermitian metric satisfying $\mathfrak{R i c}(\omega)=0$, or equivalently, by formula (4.1)

$$
\operatorname{Ric}(\omega)=\frac{\partial \partial^{*} \omega+\overline{\partial \partial}^{*} \omega}{2}
$$

The first obstruction for the existence of Levi-Civita Ricci-flat Hermitian metric is the top first Chern number: 
Corollary 5.1. Suppose $c_{1}^{\mathrm{AC}}(X)=0$, then the top intersection number $c_{1}^{n}(X)=0$. In particular, if $X$ has a Levi-Civita Ricci-flat Hermitian metric $\omega$, then $c_{1}^{n}(X)=0$.

Proof. By definition, if $c_{1}^{\mathrm{AC}}(X)=0$, then

$$
\operatorname{Ric}(\omega)=\bar{\partial} A+\partial B
$$

where $A$ is a $(1,0)$-form and $B$ is a $(0,1)$-form. Hence

$$
c_{1}^{n}(X)=\int_{X}(\operatorname{Ric}(\omega))^{n}=\int_{X}(\operatorname{Ric}(\omega))^{n-1} \wedge(\bar{\partial} A+\partial B)=0
$$

since $\operatorname{Ric}(\omega)$ is both $\partial$ and $\bar{\partial}$-closed.

Theorem 5.2. Let $X$ be a compact complex manifold. Suppose $\omega$ is a Levi-Civita Ricci-flat Hermitian metric. Then either

(1) $\kappa(X)=-\infty$; or

(2) $\kappa(X)=0$ and $(X, \omega)$ is conformally balanced with $K_{X}$ a holomorphic torsion, i.e. $K_{X}^{\otimes m}=\mathcal{O}_{X}$ for some $m \in \mathbb{Z}^{+}$.

Proof. Let $\omega_{f}=e^{f} \omega$ be the Gauduchon metric in the conformal class of $\omega$. Then by formula (4.8), the total Chern scalar curvature of $\omega_{f}$ is

$$
\int_{X} s_{f} \cdot \omega_{f}^{n}=n \int_{X} \operatorname{Ric}\left(\omega_{f}\right) \wedge \omega_{f}^{n-1}=n\left\|\bar{\partial}_{f}^{*} \omega_{f}\right\|_{\omega_{f}}^{2}
$$

since the Levi-Civita scalar curvature $s_{\mathrm{LC}}=0$. Suppose $\bar{\partial}_{f}^{*} \omega_{f} \neq 0$, then

$$
\int_{X} s_{f} \cdot \omega_{f}^{n}>0
$$

By [24, Corollary 3.3], we have $\kappa(X)=-\infty$. On the other hand, if $\bar{\partial}_{f}^{*} \omega_{f}=0$, i.e. $(X, \omega)$ is conformally balanced. Then the total Chern scalar curvature of the Gauduchon metric

$$
\int_{X} s_{f} \cdot \omega_{f}^{n}=0
$$

Then by [24, Theorem 1.4], we have $\kappa(X)=-\infty$ or $\kappa(X)=0$, and when $\kappa(X)=0, K_{X}$ is a holomorphic torsion.

Definition $5.3([4])$. Let $X$ be a compact complex manifold. $X$ is said to satisfy the $\partial \bar{\partial}-$ lemma if the following statement holds: if $\eta$ is $d$-exact, $\partial$-closed and $\bar{\partial}$-closed, it must be $\partial \bar{\partial}$-exact. In particular, on such manifolds, for any pure-type form $\varphi \in \Omega^{p, q}(X)$, if $\varphi$ is $\bar{\partial}$-closed and $\partial$-exact, then it is $\partial \bar{\partial}$-exact.

Let $\mu: \widehat{X} \rightarrow X$ be a modification between compact complex manifolds. If the $\partial \bar{\partial}$-lemma holds for $\widehat{X}$, then the $\partial \bar{\partial}$-lemma also holds for $X$. In particular, Moishezon manifolds and manifolds in Fujiki class $\mathscr{C}$ support the $\partial \bar{\partial}$-lemma. For more details, we refer to $[4,1]$ and also the references therein.

Theorem 5.4. Let $X$ be a compact complex manifold and $\omega$ be a Levi-Civita Ricci-flat Hermitian metric. If $X$ supports the $\partial \bar{\partial}-$ Lemma, then $(X, \omega)$ is conformally balanced and $K_{X}$ is unitary flat. 
Proof. By formula (4.1), we have

$$
\operatorname{Ric}(\omega)=\frac{\partial \partial^{*} \omega+\overline{\partial \partial}^{*} \omega}{2}
$$

since $\mathfrak{R i c}(\omega)=0$. Note that $\operatorname{Ric}(\omega)$ is $\partial$-closed and $\bar{\partial}$-closed, and so we have

$$
\bar{\partial} \partial \partial^{*} \omega=0
$$

Moreover, if $X$ supports the $\partial \bar{\partial}$-Lemma, then the $\bar{\partial}$-closed and $\partial$-exact $(1,1)$-form $\partial \partial^{*} \omega$ is $\partial \bar{\partial}$-exact, i.e. there exists a smooth function $\varphi$ such that

$$
\partial \partial^{*} \omega=\partial \bar{\partial} \varphi \text {. }
$$

Therefore, $\operatorname{Ric}(\omega)=\sqrt{-1} \partial \bar{\partial} F$ where $F=-\frac{\bar{\varphi}-\varphi}{2 \sqrt{-1}} \in C^{\infty}(X, \mathbb{R})$. It is obvious that the Hermitian metric $e^{\frac{F}{n}} \omega$ is Chern Ricci-flat, i.e. $K_{X}$ is unitary flat. Moreover, for any Gauduchon metric $\omega_{G}$ on $X$, we have

$$
\int_{X} \operatorname{Ric}\left(\omega_{G}\right) \wedge \omega_{G}^{n-1}=\int_{X} \operatorname{Ric}(\omega) \wedge \omega_{G}^{n-1}=\int_{X} \sqrt{-1} \partial \bar{\partial} F \wedge \omega_{G}^{n-1}=0 .
$$

Let $\omega_{f}=e^{f} \omega$ be the Gauduchon metric in the conformal class of $\omega$, then by (5.2), we have

$$
\int_{X} \operatorname{Ric}\left(\omega_{f}\right) \wedge \omega_{f}^{n-1}=\left\|\bar{\partial}_{f}^{*} \omega_{f}\right\|_{\omega_{f}}^{2}=0,
$$

that is $\bar{\partial}_{f}^{*} \omega_{f}=0$.

6. Classification of minimal complex surfaces with Levi-Civita Ricci-flat metrics

In this section, we investigate the Levi-Civita Ricci-flat metrics on minimal complex surfaces and prove Theorem 1.2.

Theorem 6.1. Let $X$ be a minimal complex surface with $\kappa(X) \geq 0$. Suppose $X$ admits a Levi-Civita Ricci-flat Hermitian metric $\omega$. Then $X$ is Kähler surface of Calabi-Yau type, i.e. $X$ is exactly one of the following

(1) a Enriques surface;

(2) a bi-elliptic surface;

(3) a K3 surface;

(4) a torus.

Proof. By Theorem 5.2, we have $\kappa(X) \leq 0$. Hence, we only need to consider minimal surfaces with $\kappa(X)=0$. By the Kodaira-Enriques' classification of minimal surfaces (e.g. [2, p. 244]), a minimal surface with $\kappa(X)=0$ has torsion canonical line bundle $K_{X}$, i.e. $K_{X}^{\otimes m}=\mathcal{O}_{X}$ for some $m \in \mathbb{Z}^{+}$. Hence, there exists a Hermitian metric $\omega_{0}$ with $\operatorname{Ric}\left(\omega_{0}\right)=0$. Let $\omega$ be the Hermitian metric with $\mathfrak{R i c}(\omega)=0$ and $\omega_{f}=e^{f} \omega$ be the Gauduchon metric in the conformal class of $\omega$. Then

$$
\int_{X} \operatorname{Ric}\left(\omega_{f}\right) \wedge \omega_{f}=\int_{X} \operatorname{Ric}\left(\omega_{0}\right) \wedge \omega_{f}=0
$$

Hence, by (5.2), we obtain $\bar{\partial}_{f}^{*} \omega_{f}=0$. Since $\operatorname{dim} X=2$, we have $d \omega_{f}=0$, i.e. $X$ is a Kähler surface. According to the Kodaira-Enriques' classification, $X$ is either an Enriques surface, a 
bi-elliptic surface, a K3 surface or a torus. All these surfaces are Kähler surfaces of Calabi-Yau type, and all Kähler Calabi-Yau metrics are Levi-Civita Ricci-flat.

Remark 6.2. The Hermitian metric $\omega$ with $\mathfrak{R i c}(\omega)=0$ in Theorem 6.1 is not necessarily Kähler. Indeed, let $\omega_{\mathrm{CY}}$ be a Calabi-Yau Kähler metric on $X$. Then for any non constant smooth function $f \in C^{\infty}(X, \mathbb{R})$, we can construct a non-Kähler Levi-Civita Ricci-flat metric. By Yau's theorem, there exists a Kähler metric $\omega_{0}$ such that

$$
\omega_{0}^{2}=e^{-f} \omega_{\mathrm{CY}}^{2} .
$$

Let $\omega=e^{f} \omega_{0}$. Then $\omega$ is a non-Kähler metric with Levi-Civita Ricci-flat curvature. Indeed,

$$
\begin{aligned}
\mathfrak{R i c}(\omega) & =\operatorname{Ric}(\omega)-\frac{\partial \partial^{*} \omega+\overline{\partial \partial}^{*} \omega}{2} \\
& =\operatorname{Ric}\left(\omega_{0}\right)-2 \sqrt{-1} \partial \bar{\partial} f-\frac{\partial \bar{\partial}_{0}^{*} \omega_{0}+\overline{\partial \partial}_{0}^{*} \omega_{0}}{2}+\sqrt{-1} \partial \bar{\partial} f \\
& =\operatorname{Ric}\left(\omega_{\mathrm{CY}}\right)+\sqrt{-1} \partial \bar{\partial} f-2 \sqrt{-1} \partial \bar{\partial} f-\frac{\partial \bar{\partial}_{0}^{*} \omega_{0}+\overline{\partial \partial}_{0}^{*} \omega_{0}}{2}+\sqrt{-1} \partial \bar{\partial} f \\
& =0
\end{aligned}
$$

where we use Lemma 4.2 in the second identity.

Theorem 6.3. Let $X$ be a minimal complex surface with $\kappa(X)=-\infty$. Suppose $X$ admits a Levi-Civita Ricci-flat Hermitian metric $\omega$. Then $X$ is a Hopf surface.

Proof. According to the Kodaira-Enriques' classification [2, p. 244], $X$ is one of the following

(1) a minimal rational surface;

(2) a minimal surface of class VII;

(3) a ruled surface of genus $g \geq 1$.

Suppose $X$ is a Kähler surface, i.e. $X$ is a minimal rational surface or a ruled surface of genus $g \geq 1$. Then the $\partial \bar{\partial}$-Lemma holds on $X$. Hence by Theorem $5.4, K_{X}$ is unitary flat and $\kappa(X)=0$ which a is contradiction. Hence $X$ is non-Kähler, i.e. $X$ is a minimal surface of class VII. By Corollary 7.1, Theorem 7.2 and Theorem 7.3 in the next section, we complete the proof.

Corollary 6.4. Let $X$ be a Kodaira surface or an Inoue surface. Then

$$
c_{1}^{\mathrm{BC}}(X)=c_{1}^{\mathrm{AC}}(X)=0 .
$$

However, $X$ does not admit a Levi-Civita Ricci-flat Hermitian metric.

Proof. It is well-known ([2, p. 244]) that a Kodaira surface is a non-Kähler surface with torsion canonical line bundle $K_{X}$. Hence $c_{1}^{\mathrm{BC}}(X)=c_{1}^{\mathrm{AC}}(X)=0$ and $\kappa(X)=0$. By Theorem 6.1, it has no Levi-Civita Ricci-flat Hermitian metric. For an Inoue surface, one has $b_{2}(X)=0$. Hence, $c_{1}(X)=c_{1}^{\mathrm{AC}}(X)=0$. 
7. The Levi-Civita Ricci-flat metrics on minimal surfaces of class VII

A class VII surface is a minimal compact complex surface with $b_{1}=1$ and $\kappa(X)=-\infty$. There are three classes of them

(1) Hopf surfaces: whose universal cover is $\mathbb{C}^{2}-\{0\}$, or equivalently a class VII surface with $b_{2}=0$ and contains a curve;

(2) Inoue surfaces: a class VII surface has $b_{2}=0$ and contains no curves;

(3) all class VII surfaces with $b_{2}>0$.

Corollary 7.1. On VII surfaces with $b_{2}>0$, there is no Levi-Civita Ricci-flat Hermitian metrics.

Proof. It is well-known that on minimal VII surfaces we have

$$
c_{1}^{2}(X)=-b_{2} .
$$

Corollary 7.1 follows from Corollary 5.1.

7.1. Inoue surfaces. It is well-known ([11]) that an Inoue surface is a quotient of $\mathbb{H} \times \mathbb{C}$ by a properly discontinuous group of affine transformations where $\mathbb{H}$ is the upper half-plane. There are three types of Inoue surfaces:

(1) Inoue surfaces $S_{M}$. Let $M$ be a matrix in $\mathrm{SL}_{3}(\mathbb{Z})$ admitting one real eigenvalue $\alpha>1$ and two complex conjugate eigenvalues $\beta \neq \bar{\beta}$. Let $\left(a_{1}, a_{2}, a_{3}\right)$ be a real eigenvector of $M$ corresponding to $\alpha$ and let $\left(b_{1}, b_{2}, b_{3}\right)$ be an eigenvector of $M$ corresponding to $\beta$. Then $X=S_{M}$ is the quotient of $\mathbb{H} \times \mathbb{C}$ by the group of affine automorphisms generated by

$$
\begin{gathered}
g_{0}(w, z)=(\alpha w, \beta z), \\
g_{i}(w, z)=\left(w+a_{i}, z+b_{i}\right), \quad i=1,2,3 .
\end{gathered}
$$

(2) Inoue surfaces $X=S_{N, p, q, r ; t}^{+}$are defined as the quotient of $\mathbb{H} \times \mathbb{C}$ by the group of affine automorphisms generated by

$$
\begin{gathered}
g_{0}(w, z)=(\alpha w, z+t), \\
g_{i}(w, z)=\left(w+a_{i}, z+b_{i} w+c_{i}\right), \quad i=1,2 \\
g_{3}(w, z)=\left(w, z+\frac{b_{1} a_{2}-b_{2} a_{1}}{r}\right),
\end{gathered}
$$

where $\left(a_{1}, a_{2}\right)$ and $\left(b_{1}, b_{2}\right)$ are the eigenvectors of some matrix $N \in \mathrm{SL}_{2}(\mathbb{Z})$ admitting real eigenvalues $\alpha>1, \alpha^{-1}$. Moreover $t \in \mathbb{C}$ and $p, q, r(r \neq 0)$ are integers, and $\left(c_{1}, c_{2}\right)$ depends on $\left(a_{i}, b_{i}\right), p, q, r$ (see [11]).

(3) Inoue surfaces $X=S_{N, p, q, r ; t}^{-}$have unramified double cover which are Inoue surfaces of type $S_{N, p, q, r ; t}^{+}$.

Theorem 7.2. On Inoue surfaces, there is no Levi-Civita Ricci-flat Hermitian metrics.

Proof. Suppose $\omega$ is a Levi-Civita Ricci-flat Hermitian metric on the Inoue surface $X$. Let $\omega_{f}=e^{f} \omega$ be the Gauduchon metric in the conformal class of $\omega$, then by formula (4.8), the total Chern scalar curvature of $\omega_{f}$ is

$$
\int_{X} s_{f} \cdot \omega_{f}^{2}=2 \int_{X} \operatorname{Ric}\left(\omega_{f}\right) \wedge \omega_{f}=2\left\|\bar{\partial}_{f}^{*} \omega_{f}\right\|_{\omega_{f}}^{2} \geq 0 .
$$


We shall show that on each Inoue surface, there exists a smooth Gauduchon metric with non-positive but not identically zero first Chern-Ricci curvature. Indeed, let $(w, z) \in \mathbb{H} \times \mathbb{C}$ be the holomorphic coordinates, then by the precise definition of each Inoue surface (see also $[5,6,18])$, we know the form

$$
\sigma=\frac{d w \wedge d z}{\operatorname{Im}(w)}
$$

descends to a smooth nowhere vanishing $(2,0)$ form on $X$, i.e. $\sigma \in \Gamma\left(X, K_{X}\right)$. Then it induces a smooth Hermitian metric $h$ on $K_{X}$ given by $h(\sigma, \sigma)=1$. In the holomorphic frame $e=d w \wedge d z$ of $K_{X}$, we have

$$
h=h(e, e)=[\operatorname{Im}(w)]^{2} .
$$

It also induces a Hermitian metric $h^{-1}$ on $K_{X}^{-1}$, and the curvature of $h^{-1}$ is

$$
-\sqrt{-1} \partial \bar{\partial} \log h^{-1}=\sqrt{-1} \partial \bar{\partial} \log [\operatorname{Im}(w)]^{2}=-\frac{\sqrt{-1}}{2} \frac{d w \wedge d \bar{w}}{[\operatorname{Im}(w)]^{2}}
$$

which also represents $c_{1}^{\mathrm{BC}}(X)$. By Theorem [17, Theorem 1.3], there exists a Gauduchon metric $\omega_{G}$ with

$$
\operatorname{Ric}\left(\omega_{G}\right)=-\frac{\sqrt{-1}}{2} \frac{d w \wedge d \bar{w}}{[\operatorname{Im}(w)]^{2}} \leq 0
$$

Hence, for any Gauduchon metric $\omega$, one has

$$
\int_{X} \operatorname{Ric}(\omega) \wedge \omega=\int_{X} \operatorname{Ric}\left(\omega_{G}\right) \wedge \omega<0
$$

which is a contradiction to (7.1).

7.2. Hopf manifolds. Let's recall an example in [13, 14, Section 6]. Let $X=\mathbb{S}^{2 n-1} \times \mathbb{S}^{1}$ be the standard $n$-dimensional $(n \geq 2)$ Hopf manifold. It is diffeomorphic to $\mathbb{C}^{n}-\{0\} / G$ where $G$ is cyclic group generated by the transformation $z \rightarrow \frac{1}{2} z$. It has a naturally induced metric $\omega_{0}$ given by

$$
\omega_{0}=\sqrt{-1} \frac{\delta_{i \bar{j}}}{|z|^{2}} d z^{i} \wedge d \bar{z}^{j}
$$

We present a straightforward computation to show (c.f. [14, Theorem 6.2]):

Theorem 7.3. The perturbed metric

$$
\omega_{g}=\omega_{0}-\frac{1}{n} \cdot \sqrt{-1} \partial \bar{\partial} \log |z|^{2} .
$$

is Levi-Civita Ricci-flat, i.e. $\mathfrak{R i c}\left(\omega_{g}\right)=0$.

Proof. If we write $\omega_{g}=\sqrt{-1} g_{i j} d z^{i} \wedge d \bar{z}^{j}$, then

$$
g_{i \bar{j}}=\frac{1}{|z|^{2}}\left(\frac{n-1}{n} \delta_{i j}+\frac{\bar{z}^{i} z^{j}}{n|z|^{2}}\right), \quad \text { and } \quad g^{i \bar{j}}=|z|^{2}\left(\frac{n \delta_{i \bar{j}}}{n-1}-\frac{z^{i} \bar{z}^{j}}{(n-1)|z|^{2}}\right) .
$$

Let $\partial^{*}$ and $\bar{\partial}^{*}$ be the adjoint operators with respect to $\omega_{g}$ and $\Lambda$ is the dual operator of $\omega_{g} \wedge \bullet$. A straightforward computation ([14, Lemma 3.3]) shows

$$
\bar{\partial}^{*} \omega_{g}=\sqrt{-1} \Lambda \partial \omega_{g} .
$$


Note that

Hence, we obtain

$$
\partial \omega_{g}=\partial \omega_{0}=-\frac{\sqrt{-1} \delta_{i \bar{j}} \bar{z}^{k}}{|z|^{4}} d z^{k} \wedge d z^{i} \wedge d \bar{z}^{j}
$$

$$
\begin{aligned}
\bar{\partial}^{*} \omega_{g}=\sqrt{-1} \Lambda \partial \omega_{g} & =\sqrt{-1} g^{k \bar{q}} \frac{\delta_{i \bar{q}} \bar{z}^{k}}{|z|^{4}} d z^{i}-\sqrt{-1} g^{i \bar{q}} \frac{\delta_{i \bar{q}} \bar{z}^{k}}{|z|^{4}} d z^{k} \\
& =\sqrt{-1} \frac{\sum_{k} g^{k i} \bar{z}^{k}}{|z|^{4}} d z^{i}-\sqrt{-1} \frac{\sum_{q} g^{q \bar{q}} \bar{z}^{k}}{|z|^{4}} d z^{k}
\end{aligned}
$$

On the other hand,

$$
\sum_{k} g^{k \bar{i}} \bar{z}^{k}=|z|^{2} \bar{z}^{i} \quad \text { and } \quad \sum_{q} g^{q \bar{q}} \bar{z}^{k}=(n+1)|z|^{2} \bar{z}^{k} .
$$

Hence, we have

$$
\bar{\partial}^{*} \omega_{g}=\sqrt{-1} n \frac{\bar{z}^{k} d z^{k}}{|z|^{2}}=-n \sqrt{-1} \partial \log |z|^{2}
$$

and

$$
\overline{\partial \partial}^{*} \omega_{g}=n \sqrt{-1} \partial \bar{\partial} \log |z|^{2} .
$$

Therefore

$$
\frac{\partial \partial^{*} \omega_{g}+\overline{\partial \partial}^{*} \omega_{g}}{2}=\sqrt{-1} n \partial \bar{\partial} \log |z|^{2} .
$$

A direct computation shows $\operatorname{det}\left(g_{i \bar{j}}\right)=(1+\lambda)^{n-1}|z|^{-2 n}$, and we have

$$
\operatorname{Ric}\left(\omega_{g}\right)=-\sqrt{-1} \partial \bar{\partial} \log \operatorname{det}(g)=n \cdot \sqrt{-1} \partial \bar{\partial} \log |z|^{2} .
$$

By Theorem 4.1,

$$
\mathfrak{R i c}\left(\omega_{g}\right)=\operatorname{Ric}\left(\omega_{g}\right)-\frac{\partial \partial^{*} \omega_{g}+\overline{\partial \partial}^{*} \omega_{g}}{2}=0
$$

Remark 7.4. In this example, we construct a solution to the Levi-Civita Ricci-flat equation on Hopf manifolds. It is natural to ask whether there are more solutions. We expect there are theoretical approaches on the existence of Levi-Civita Ricci-flat metrics on all Hopf manifolds.

\section{References}

[1] Angella, D.; Tomassini, A. On the $\partial \bar{\partial}-$ lemma and Bott-Chern cohomology. Invent. Math. 192 (2013), no. 1, 71-81.

[2] Barth, W.; Hulek, K.; Peters, C.; Van de Ven, A. Compact complex surfaces. Ergebnisse der Mathematik und ihrer Grenzgebiete. 3. Folge. A Series of Modern Surveys in Mathematics. Springer-Verlag, Berlin, 2004.

[3] Chiose, I.; Rasdeaconu, R.; Suvaina, I. Balanced metrics on uniruled manifolds. arXiv:1408.4769. To appear in Comm. Anal. Geom.

[4] Deligne, P.; Griffiths, P.; Morgan, J.; Sullivan, D. Real homotopy theory of Kähler manifolds. Invent. Math. 29 (1975), no. 3, 245-274.

[5] Demailly, J.-P.; Peternell, T.; Schneider, M. Compact complex manifolds with numerically effective tangent bundles. J. Algebraic Geom. 3 (1994), no. 2, 295-345.

[6] Fang, S.-W.; Tosatti, V.; Weinkove, B. and Zheng, T. Inoue surfaces and the Chern-Ricci flow. J. Funct. Anal. 271 (2016), 3162-3185. 
[7] Fu, J.-X. On non-Kähler Calabi-Yau threefolds with balanced metrics. Proceedings of the International Congress of Mathematicians. Volume II, 705-716, Hindustan Book Agency, New Delhi, 2010.

[8] Fu, J.- X.; Yau, S.-T. The theory of superstring with flux on non-Kähler manifolds and the complex Monge-Ampére equation. J. Differential Geom. 78 (2008), no. 3, 369-428.

[9] Fu, J.- X; Li, J.; Yau, S.-T. Constructing balanced metrics on some families of non-Kähler Calabi-Yau threefolds. J. Differential Geom. 90 (2012), no. 1,81-129.

[10] Gauduchon, P. Fibrés hermitiens à endomorphisme de Ricci non-négatif, Bull. Soc. Math. France 105 1977), 113-140.

[11] Inoue, M. On surfaces of type VII $_{0}$, Inv. Math., 24 (1974), 269-310.

[12] Li, Yi. A priori estimates for Donaldsons equation over compact Hermitian manifolds. Calc. Var. Partial Differential Equations, 50(2014), no. 3-4, 867-882.

[13] Liu, K.-F.; Yang, X.-K. Geometry of Hermitian manifolds. Internat. J. Math. 23 (2012) 40pp.

[14] Liu, K.-F.; Yang, X.-K. Ricci curvatures on Hermitian manifolds.Trans. Amer. Math. Soc. 369 (2017), 5157-5196.

[15] Streets, J.; Tian, G. A parabolic flow of pluriclosed metrics, Int. Math. Res. Not. 2010, no. 16, 3101-3133.

[16] Streets, J.; Tian, G. Regularity results for pluriclosed flow, Geom. Topol. 17 (2013), no. 4, 2389-2429.

[17] Székelyhidi, G.; Tosatti, V.; Weinkove, B. Gauduchon metrics with prescribed volume form. arXiv:1503.04491.

[18] Teleman, A. The pseudo-effective cone of a non-Kählerian surface and applications. Math. Ann. 335(2006), 965-989.

[19] Tosatti, V. Non-Kähler Calabi-Yau manifolds. Contemp. Math. 644(2015), 261-277.

[20] Tosatti, V.; Weinkove, B. On the evolution of a Hermitian metric by its Chern-Ricci form. J. Differential Geom. 99 (2015), no.1, 125-163.

[21] Tosatti, V.; Weinkove, B. The Monge-Ampère equation for $(n-1)$-plurisubharmonic functions on a compact Kähler manifold. J. Amer. Math. Soc. 30 (2017), no.2, 311-346.

[22] Yang, X.-K. Hermitian manifolds with semi-positive holomorphic sectional curvature. Math. Res. Lett. 23 (2016), no.3, 939-952.

[23] Yang, X.-K. The Chern-Ricci flow and holomorphic bisectional curvature. Sci. China Math. 59 (2016), 2199-2204.

[24] Yang, X.-K. Scalar curvature on compact complex manifolds. arXiv:1705.02672

[25] Yau, S.-T. On the curvature of compact Hermitian manifolds. Invent. Math. 25 (1974), 213-239.

[26] Yau, S.-T. On the Ricci curvature of a compact Kähler manifold and the complex Monge-Ampère equation, I, Comm. Pure Appl. Math. 31 (1978), 339-411.

Department of Mathematics, Capital Normal University, Beijing, 100048, China

Department of Mathematics, University of California at Los Angeles, California 90095

E-mail address: liu@math.ucla.edu

Address of Xiaokui Yang: Morningside Center of Mathematics, Institute of Mathematics, Hua Loo-Keng Key Laboratory of Mathematics, Academy of Mathematics and Systems Science, Chinese Academy of Sciences, Beijing, 100190, China.

E-mail address: xkyang@ams.ac.cn 